\section{Image Noise Induced Errors in Camera Positioning}

\author{
Graziano Chesi, Senior Member, IEEE, and \\ Y.S. Hung, Senior Member, IEEE
}

\begin{abstract}
The problem of evaluating worst-case camera positioning error induced by unknown-but-bounded (UBB) image noise for a given object-camera configuration is considered. Specifically, it is shown that upper bounds to the rotation and translation worst-case error for a certain image noise intensity can be obtained through convex optimizations. These upper bounds, contrary to lower bounds provided by standard optimization tools, allow one to design robust visual servo systems.
\end{abstract}

Index Terms-Visual servoing, image noise, positioning accuracy, convex optimization.

\section{INTRODUCTION}

A fundamental problem in robotics consists of positioning a six degrees of freedom (dof) end-effector with respect to an object. An emerging approach to deal with this problem consists of using the view of a set of cameras as feedback in a control system. The motivation is to exploit simultaneously the benefits of closed-loop control and visual sensor to improve positioning accuracy and robustness. Typically, one camera mounted on the robot endeffector is used, in the so-called "eye-in-hand" configuration. The approach can be described as follows: First, the camera is located in a desired position with respect to the object. The view of the camera in this position, called desired view, is hence stored. Then, the camera is moved to any other unknown position of the scene. The target is to reposition the camera in the desired position by exploiting the difference between the actual view of the camera, called current view, and the desired view. This approach is known in the literature as teaching-by-showing.

Several methods have been developed to position the camera in the teaching-by-showing approach (see, for example, [8]). The classic methods are image-based visual servoing, position-based visual servoing, and 2 1/2D visual servoing (see [15], [17], [11], respectively). Then, other visual servoing methods are based on partitioning strategies [7], [5], [14], navigation functions [6], pathplanning techniques [18], [12], transformations invariant with respect to the intrinsic parameters [10], image moments [16], and generations of circular-like trajectories [4].

In all these methods, the target of positioning the camera is transformed into the target of matching the desired view with the current view. Therefore, the accuracy of the camera positioning is strongly affected by image noise (an analysis of the effect of image noise on the control law of position-based visual servoing and $21 / 2$ D visual servoing has been proposed in [9]). Clearly, it would be useful to quantify the errors induced by the image noise on the camera positioning. However, such a quantification amounts to solving a nonconvex maximization problem, which is very hard to solve due to the presence of local maxima that prevent one from establishing the worst-case positioning error.

- The authors are with the Department of Electrical and Electronic Engineering, University of Hong Kong, Pokfulam Road, Hong Kong. E-mail: \{chesi, yshung\}@eee.hku.hk.

Manuscript received 3 May 2006; revised 12 Jan. 2007; accepted 24 Jan. 2007; published online 13 June 2007.

Recommended for acceptance by $C$. Taylor.

For information on obtaining reprints of this article, please send e-mail to: tpami@computer.org, and reference IEEECS Log Number TPAMI-0344-0506.
In this paper, some new conditions to evaluate these errors for a given object-camera configuration are proposed through convex optimizations. Specifically, it is shown that upper bounds to the rotation and translation worst-case error for a certain unknown-butbounded (UBB) image noise intensity can be obtained through eigenvalue problems (EVPs) which are convex optimizations constrained by linear matrix inequalities (LMIs). This is achieved, first, by parameterizing the rotation matrix through the Cayley parameter, and second, by introducing suitable polynomial relaxations. The advantage of the proposed technique with respect to standard optimization tools for solving the problem is obvious: While these tools provide lower bounds only of the worst-case error due to the presence of local maxima, the proposed technique provides upper bounds. It is clear that, in order to realize a robust visual servo system, upper bounds of the worst-case error are required.

The paper is organized as follows: Section 2 introduces the problem formulation. Section 3 presents the proposed technique to compute upper bounds of the worst-case error. Section 4 provides an illustrative example. Finally, Section 5 concludes with some final remarks.

\section{Problem Formulation}

The notation is as follows: $\mathbf{I}_{n}$ is the identity matrix $n \times n, \mathbf{0}_{n}$ the null vector $n \times 1, \mathbf{e}_{i}$ the $i$ th column of $\mathbf{I}_{3}, S O(3)$ the set of all $3 \times 3$ rotation matrices, $[\mathbf{v}]_{\times} \in \mathbb{R}^{3 \times 3}$ the skew-symmetric matrix of $\mathbf{v} \in \mathbb{R}^{3}$, and $\|\mathbf{w}\|$ (respectively, $\|\mathbf{w}\|_{\infty}$ ) the euclidean (respectively, infinity) norm of vector $\mathbf{w}$.

Let $\mathcal{F}^{*}$ be the absolute reference frame and the desired camera frame. The $i$ th $3 \mathrm{D}$ point $\mathbf{q}_{i}=\left[x_{i} ; y_{i} ; z_{i}\right]$ projects on $\mathcal{F}^{*}$ at the point $\mathbf{m}_{i}^{*}$ defined by $d_{i}^{*} \mathbf{m}_{i}^{*}=\mathbf{I}_{3} \mathbf{q}_{i}+\mathbf{0}_{3}$, where $d_{i}^{*}$ is the depth with respect to $\mathcal{F}^{*}$. Let $\mathcal{F}$ be the current camera frame and let $\mathbf{R}$ and $\mathbf{t}$ be, respectively, the rotation and translation of $\mathcal{F}$ with respect to $\mathcal{F}^{*}$. The 3D point $\mathbf{q}_{i}$ projects on $\mathcal{F}$ at the point $\mathbf{m}_{i}$ defined by $d_{i} \mathbf{m}_{i}=\mathbf{R} \mathbf{q}_{i}+\mathbf{t}$, where $d_{i}$ is the depth with respect to $\mathcal{F}$. The frame points $\mathbf{m}_{i}^{*}$ and $\mathbf{m}_{i}$ project on the camera image plane at points $\mathbf{p}_{i}^{*}=\mathbf{K m}_{i}^{*}$ and $\mathbf{p}_{i}=\mathbf{K m}_{i}$, respectively, where $\mathbf{K}$ is the upper-triangular intrinsic parameters matrix. The points $\mathbf{p}_{i}^{*}=$ $\left[p_{i, x}^{*} ; p_{i, y}^{*} ; 1\right]$ and $\mathbf{p}_{i}=\left[p_{i, x} ; p_{i, y} ; 1\right], i=1, \ldots, n$, are gathered in the vector $\mathbf{p}^{*}=\left[p_{1, x}^{*} ; p_{1, y}^{*} ; \ldots ; p_{n, x}^{*} ; p_{n, y}^{*}\right]$ and $\mathbf{p}=\left[p_{1, x} ; p_{1, y} ; \ldots ; p_{n, x} ; p_{n, y}\right]$.

Fig. 1 illustrates the teaching-by-showing approach in the absence of image noise: The camera is first located in the desired position (Figs. 1a and 1b), then moved to any other position (Figs. 1c and 1d), then the visual servoing steers the camera from the current position to the desired position by exploiting the current and desired view of the object (Figs. 1e and 1f), and, finally, the camera motion ends when the current view matches the desired view (Figs. 1g and 1h).

However, in the presence of image noise as in real cases, the current view may never match the desired view, and even in the case of perfect matching, perfect positioning is not ensured. In fact, let $\hat{\mathbf{p}}^{*}$ and $\hat{\mathbf{p}}$ be the estimates of $\mathbf{p}^{*}$ and $\mathbf{p}$ affected by image noise,

$$
\begin{cases}\hat{\mathbf{p}}^{*}=\mathbf{p}^{*}+\mathbf{n}^{*}, & \left\|\mathbf{n}^{*}\right\|_{\infty}<\delta_{N} \\ \hat{\mathbf{p}}=\mathbf{p}+\mathbf{n}, & \|\mathbf{n}\|_{\infty}<\delta_{N}\end{cases}
$$

where $\mathbf{n}^{*}$ and $\mathbf{n}$ are the UBB image noise vectors affecting the acquisition of the desired and current view respectively, and $\delta_{N}$ denotes the noise intensity (which includes image quantization, lighting, features extraction, etc.). Then, it clearly follows that

$$
\left\|\hat{\mathbf{p}}-\hat{\mathbf{p}}^{*}\right\|_{\infty}<\delta_{S} \quad \text { guarantees only }\left\|\mathbf{p}-\mathbf{p}^{*}\right\|_{\infty}<\delta_{S}+2 \delta_{N},
$$



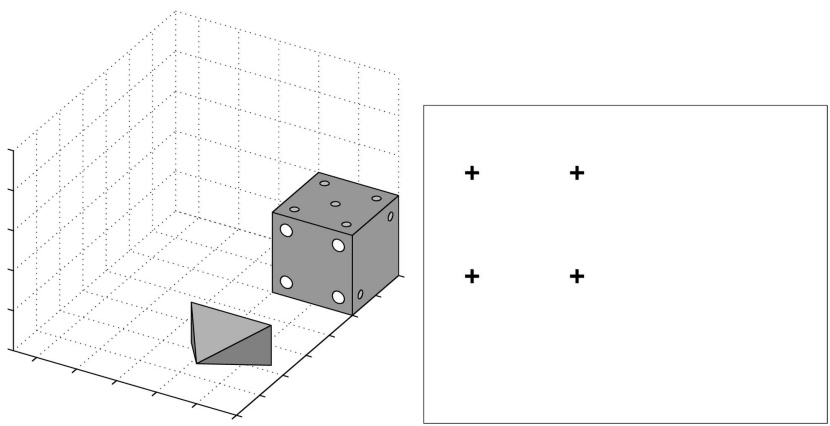

(a)

(b)

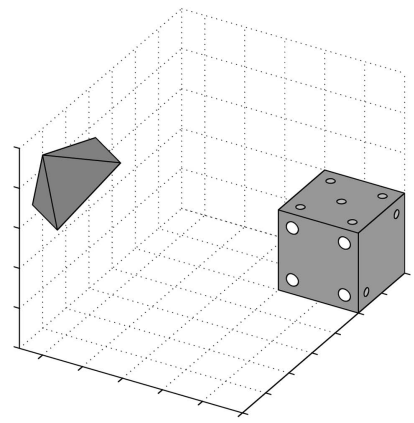

(c)

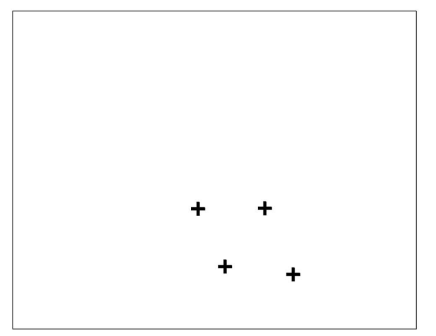

(d)

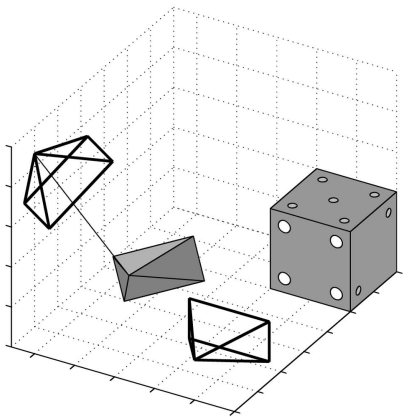

(e)

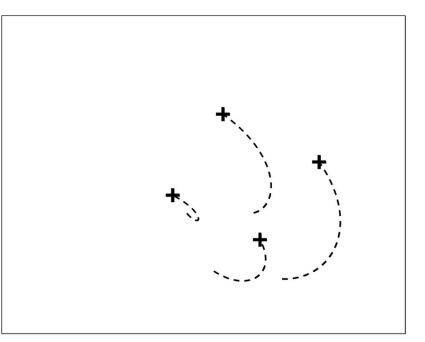

(f)

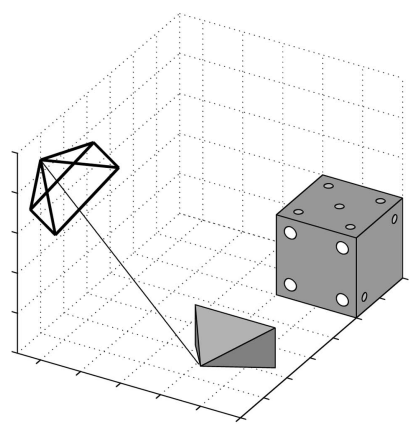

(g)

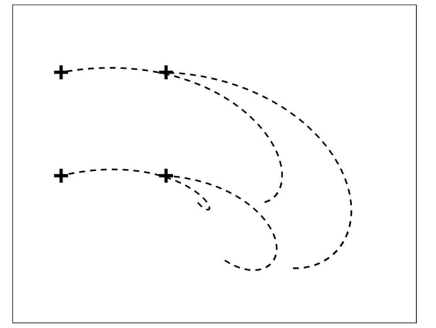

(h)

Fig. 1. Teaching-by-showing approach: (a), (c), (e), and (g) scene and (b), (d), (f), and $(h)$ corresponding camera view.

where $\left\|\hat{\mathbf{p}}-\hat{\mathbf{p}}^{*}\right\|_{\infty}<\delta_{S}$ is the visual servoing goal condition for a chosen threshold $\delta_{S}$. This means that there is no way to guarantee that the real image error $\left\|\mathbf{p}-\mathbf{p}^{*}\right\|_{\infty}$ converges to a value smaller than $2 \delta_{N}$ and, hence, that the positioning error converges to zero.

Therefore, in this paper, we consider the problem of computing, for a given object-camera configuration, upper bounds of the worst-case positioning errors

$$
e_{R}=\sup _{\mathbf{R} \in S O(3), \mathbf{t} \in \mathbb{R}^{3}:\left\|\mathbf{p}-\mathbf{p}^{*}\right\|_{\infty}<\delta} \theta
$$

(worst-case rotation error)

and

$$
\begin{array}{r}
e_{T}=\sup _{\mathbf{R} \in S O(3), \mathbf{t} \in \mathbb{R}^{3}:\left\|\mathbf{p}-\mathbf{p}^{*}\right\|_{\infty}<\delta}\|\mathbf{t}\| \\
\text { (worst-case translation error), }
\end{array}
$$

where $\delta=\delta_{S}+2 \delta_{N}$ is the maximum image error, and $\theta \in[0, \pi]$ is the rotation angle of $\mathbf{R}$ in the exponential coordinates, i.e., $\mathbf{R}=$ $e^{[\theta \mathbf{u}]_{\times}}$for a rotation axis $\|\mathbf{u}\| \in \mathbb{R}^{3}$ with $\|\mathbf{u}\|=1$.

Let us observe that $e_{R}$ and $e_{T}$ depend on the number $n$ of image points since, by adding image points keeping $\delta$ constant, one reduces the set of feasible $\mathbf{R}, \mathbf{t}$ in the optimizations (3) and (4).

\section{UPPER BOUNDS COMPUTATION}

The first step to derive convex conditions consists of parameterizing the rotation matrix through the Cayley parameter [13]. Specifically, let us introduce the function

$$
\boldsymbol{\Gamma}(\mathbf{a})=\left(\mathbf{I}_{3}-[\mathbf{a}]_{\mathrm{x}}\right)^{-1}\left(\mathbf{I}_{3}+[\mathbf{a}]_{\mathrm{x}}\right),
$$

where $\mathbf{a} \in \mathbb{R}^{3}$ is the Cayley parameter. It turns out that $\boldsymbol{\Gamma}(\mathbf{a}) \in$ $S O(3)$ for all a. Moreover, for all $\mathbf{R} \in S O(3)$ there exists a (possibly unbounded) such that $\mathbf{R}=\boldsymbol{\Gamma}(\mathbf{a})$. The Cayley parameter of $\mathbf{R}$ is related to the exponential coordinates of $\mathbf{R}$ by

$$
\begin{aligned}
\|\mathbf{a}\| & =\tan \frac{\theta}{2}, \\
\frac{\mathbf{a}}{\|\mathbf{a}\|} & =\mathbf{u} .
\end{aligned}
$$

Therefore, (3) and (4) can be rewritten as

$$
\begin{aligned}
& e_{R}=2 \arctan \sqrt{\rho_{R}}, \\
& \rho_{R}=\sup _{\mathbf{a} \in \mathbb{R}^{3}, \mathbf{t} \in \mathbb{R}^{3}:\left\|\mathbf{p}-\mathbf{p}^{*}\right\|_{\infty}<\delta}\|\mathbf{a}\|^{2}
\end{aligned}
$$

and

$$
\begin{aligned}
& e_{T}=\sqrt{\rho_{T}}, \\
& \rho_{T}=\sup _{\mathbf{a} \in \mathbb{R}^{3}, \mathbf{t} \in \mathbb{R}^{3}:\left\|\mathbf{p}-\mathbf{p}^{*}\right\|_{\infty}<\delta}\|\mathbf{t}\|^{2} .
\end{aligned}
$$

Consider now the image constraint $\left\|\mathbf{p}-\mathbf{p}^{*}\right\|_{\infty}<\delta$ in the computations of $\rho_{R}$ and $\rho_{T}$ in (9), (10), and (11). For the $i$ th point, we have

$$
\mathbf{p}_{i}-\mathbf{p}_{i}^{*}=\mathbf{K} \frac{\boldsymbol{\Gamma}(\mathbf{a}) \mathbf{q}_{i}+\mathbf{t}}{\mathbf{e}_{3}^{T}\left(\boldsymbol{\Gamma}(\mathbf{a}) \mathbf{q}_{i}+\mathbf{t}\right)}-\mathbf{K} \frac{\mathbf{q}_{i}}{\mathbf{e}_{3}^{T} \mathbf{q}_{i}}
$$

Let us observe that

$$
\boldsymbol{\Gamma}(\mathbf{a})=\frac{\boldsymbol{\Omega}(\mathbf{a})}{1+\|\mathbf{a}\|^{2}},
$$

where $\Omega(\mathbf{a}) \in \mathbb{R}^{3 \times 3}$ is a quadratic matrix function of $\mathbf{a}$. Hence, (12) can be rewritten as

$$
\mathbf{p}_{i}-\mathbf{p}_{i}^{*}=\mathbf{K} \frac{\boldsymbol{\Omega}(\mathbf{a}) \mathbf{q}_{i}+\mathbf{b}}{\mathbf{e}_{3}^{T}\left(\boldsymbol{\Omega}(\mathbf{a}) \mathbf{q}_{i}+\mathbf{b}\right)}-\mathbf{K} \frac{\mathbf{q}_{i}}{\mathbf{e}_{3}^{T} \mathbf{q}_{i}}
$$




$$
\mathbf{b}=\left(1+\|\mathbf{a}\|^{2}\right) \mathbf{t} .
$$

Therefore, $\left\|\mathbf{p}-\mathbf{p}^{*}\right\|_{\infty}<\delta$ if and only if

$$
g_{i, j, k}(\mathbf{c})>0 \quad \forall(i, j, k) \in \mathcal{I},
$$

where $\mathbf{c}=[\mathbf{a} ; \mathbf{b}] \in \mathbb{R}^{6}, g_{i, j, k}(\mathbf{c})$ is the polynomial

$$
\begin{aligned}
g_{i, j, k}(\mathbf{c})= & (-1)^{k}\left(\mathbf{e}_{3}^{T} \mathbf{q}_{i} \mathbf{e}_{j}^{T} \mathbf{K}\left(\boldsymbol{\Omega}(\mathbf{a}) \mathbf{q}_{i}+\mathbf{b}\right)\right. \\
& \left.-\mathbf{e}_{3}^{T}\left(\boldsymbol{\Omega}(\mathbf{a}) \mathbf{q}_{i}+\mathbf{b}\right) \mathbf{e}_{j}^{T} \mathbf{K} \mathbf{q}_{i}\right) \\
& +\delta \mathbf{e}_{3}^{T} \mathbf{q}_{i} \mathbf{e}_{3}^{T}\left(\boldsymbol{\Omega}(\mathbf{a}) \mathbf{q}_{i}+\mathbf{b}\right)
\end{aligned}
$$

and

$$
\mathcal{I}=\{(i, j, k): i=1, \ldots, n, j=1,2, k=0,1\} .
$$

The second step of our approach consists of introducing suitable polynomial relaxations in order to solve the optimizations (9), (10), and (11). Let us define the polynomials

$$
\begin{aligned}
& f_{R}(\mathbf{c})=\gamma-\|\mathbf{a}\|^{2}-\sum_{(i, j, k) \in \mathcal{I}} s_{i, j, k}(\mathbf{c}) g_{i, j, k}(\mathbf{c}) \\
& f_{T}(\mathbf{c})=\left(1+\|\mathbf{a}\|^{2}\right)^{2} \gamma-\|\mathbf{b}\|^{2}-\sum_{(i, j, k) \in \mathcal{I}} s_{i, j, k}(\mathbf{c}) g_{i, j, k}(\mathbf{c}),
\end{aligned}
$$

where $\gamma \in \mathbb{R}$ is an auxiliary scalar, and $s_{i, j, k}(\mathbf{c})$ are auxiliary polynomials. Let $2 m_{f}$ ((respectively, $\left.2 m_{s}\right)$ be the degree of $f_{R}(\mathbf{c})$ and $f_{T}(\mathbf{c})$ (respectively, $s_{i, j, k}(\mathbf{c})$ ), and let $\mathbf{c}^{[\mathrm{m}]}$ be a vector containing all monomials of degree less than or equal to $m$ in the elements of variable c. It turns out from combinatorial mathematics that the dimension of $\mathbf{c}^{[m]}$ is

$$
\sigma(m)=\frac{(m+6) !}{(m ! 6 !)}
$$

Let us express the above polynomials as

$$
\left\{\begin{aligned}
f_{R}(\mathbf{c}) & =\mathbf{c}^{\left[m_{f}\right]^{\prime}} F_{R} \mathbf{c}^{\left[m_{f}\right]} \\
f_{T}(\mathbf{c}) & =\mathbf{c}^{\left[m_{f}\right]^{\prime}} F_{T} \mathbf{c}^{\left[m_{f}\right]} \\
s_{i, j, k}(\mathbf{c}) & =\mathbf{c}^{\left[m_{s}\right]^{\prime}} S_{i, j, k} \mathbf{c}^{\left[m_{s}\right]},
\end{aligned}\right.
$$

where $F_{R}, F_{T}$, and $S_{i, j, k}$ are any symmetric matrices of suitable dimensions satisfying (21). Finally, let $L(\alpha)$ be any linear parametrization of the linear set

$$
\mathcal{L}=\left\{L=L^{\prime}: \mathbf{c}^{\left[m_{f}\right]^{\prime}} L \mathbf{c}^{\left[m_{f}\right]}=0 \forall \mathbf{c}\right\},
$$

where $\alpha$ is a free vector.

The representation (21) is known as square matricial representation (SMR) and has been introduced in [3]. In [2], simple algorithms for the computation of SMR matrices as $F_{R}, F_{T}$, and $S_{i, j, k}$ as well as the function $L(\alpha)$ are provided, and it is shown that the dimension of $\alpha$ is

$$
\tau\left(m_{f}\right)=\frac{1}{2} \sigma\left(m_{f}\right)\left(\sigma\left(m_{f}\right)+1\right)-\sigma\left(2 m_{f}\right) .
$$

In practice, one first builds the vectors $\mathbf{c}^{\left[m_{f}\right]}$ and $\mathbf{c}^{\left[m_{s}\right]}$ by freely choosing any possible permutation of the $\sigma\left(m_{f}\right)$ monomials for $\mathbf{c}^{\left[m_{f}\right]}$ and any possible permutation of the $\sigma\left(m_{s}\right)$ monomials for $\mathbf{c}^{\left[m_{s}\right]}$. Then, one introduces the free matrix variables $S_{i, j, k}$, hence defining the polynomials $s_{i, j, k}(\mathbf{c}), f_{T}(\mathbf{c})$, and $f_{R}(\mathbf{c})$. The next step is to build any possible matrix function $F_{R}$ and any possible matrix function $F_{T}$ (both affinely depending on $S_{i, j, k}$ ) through simple coefficients equalization of the equations in (21). Finally, one constructs the matrix function $L(\alpha)$ by selecting any possible parametrization of the linear set $\mathcal{L}$.

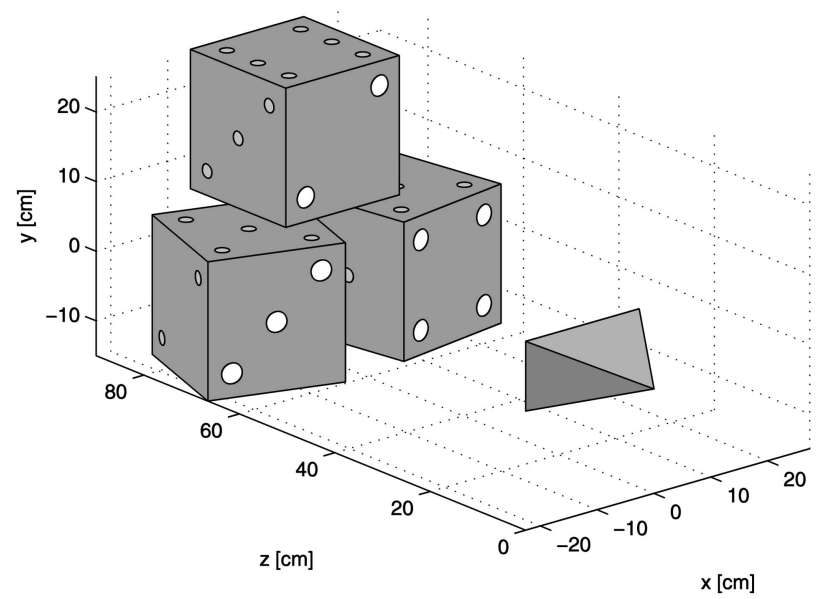

(a)

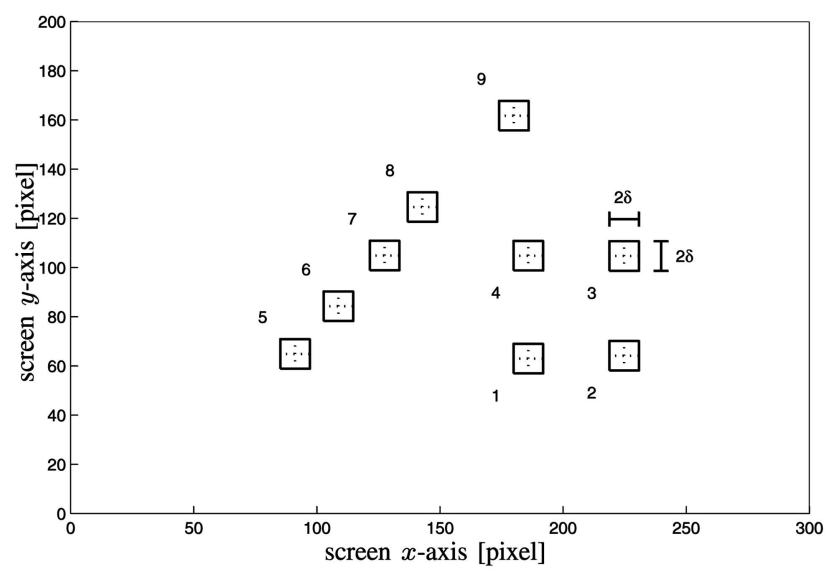

(b)

Fig. 2. Example. (a) Scene and (b) camera view. The object-camera configuration is defined by the $3 D$ points $\mathbf{q}_{1}, \ldots, \mathbf{q}_{9}$ (readable from $(\mathrm{a})$ ) and the intrinsic parameters matrix $\mathbf{K}=[200,0,150 ; 0,200,100 ; 0,0,1]$.

The following theorem shows how upper bounds of $e_{R}$ and $e_{T}$ can be obtained through convex optimization.

Theorem 1. Let us define

$$
\bar{e}_{R}=2 \arctan \sqrt{\bar{\rho}_{R}},
$$

where

$$
\begin{gathered}
\bar{\rho}_{R}=\min _{\gamma, \alpha, S_{i, j, k}} \gamma \\
\text { subject to }\left\{\begin{array}{l}
F_{R}+L(\alpha) \geq 0 \\
S_{i, j, k} \geq 0 \quad \forall(i, j, q k) \in \mathcal{I}
\end{array}\right.
\end{gathered}
$$

and

$$
\bar{e}_{T}=\sqrt{\bar{\rho}_{T}}
$$

where

$$
\begin{gathered}
\bar{\rho}_{T}=\min _{\gamma, \alpha, S_{i, j, k}} \gamma \\
\text { subject to }\left\{\begin{array}{l}
F_{T}+L(\alpha) \geq 0 \\
S_{i, j, k} \geq 0 \quad \forall(i, j, k) \in \mathcal{I} .
\end{array}\right.
\end{gathered}
$$

Then, $\bar{e}_{R} \geq e_{R}$ and $\bar{e}_{T} \geq e_{T}$.

Proof. Consider first $\bar{e}_{R}$ and suppose that the inequality constraint in (25) is satisfied for some values of $\gamma, \alpha$, and $S_{i, j, k}$. From $F_{R}+L(\alpha) \geq 0$, we obtain 


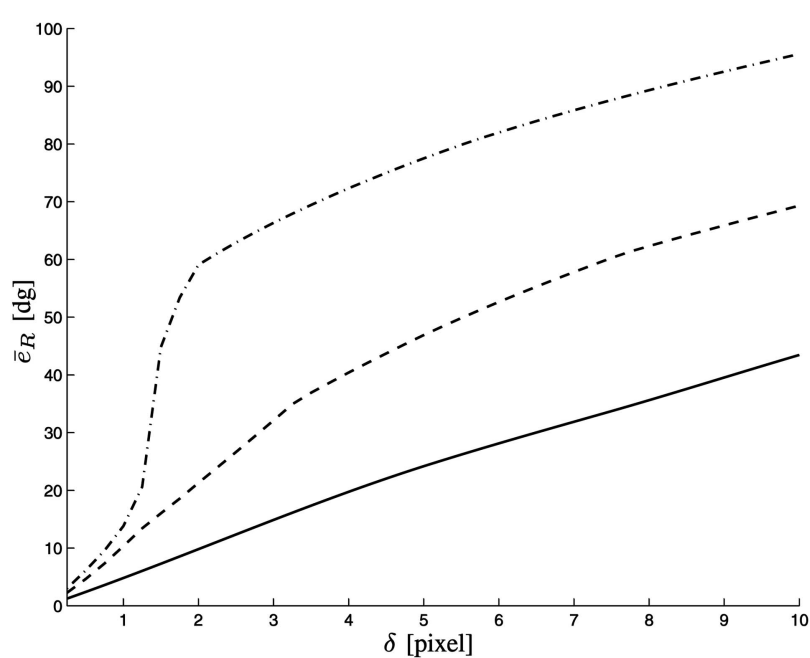

(a)

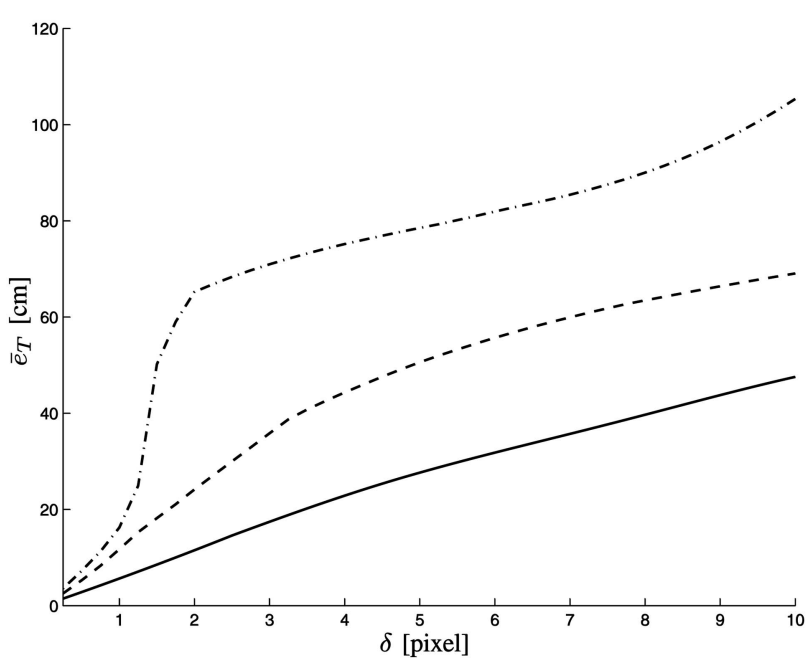

(b)

Fig. 3. Example. (a) Upper bounds $\bar{e}_{R}$ (b) and $\bar{e}_{T}$. The solid line is obtained by using all the nine points, the dashed line by using the first seven points only, and the dashdot line by using the first four points only.

$$
\begin{aligned}
0 & \leq \mathbf{c}^{\left[m_{f}\right]^{\prime}}\left(F_{R}+L(\alpha)\right) \mathbf{c}^{\left[m_{f}\right]} \\
& =\mathbf{c}^{\left[m_{f}\right]^{\prime}} F_{R} \mathbf{c}^{\left[m_{f}\right]} \\
& =f_{R}(\mathbf{c})
\end{aligned}
$$

that is, $f_{R}(\mathbf{c})$ is nonnegative for all c. Analogously, we have that $s_{i, j, k}(\mathbf{c}) \geq 0 \forall \mathbf{c}$. Consider now any c satisfying the constraint $g_{i, j, k}(\mathbf{c})>0$ for all $(i, j, k) \in \mathcal{I}$. From the positivity of $f_{R}(\mathbf{c})$, it follows that

$$
\gamma \geq\|\mathbf{a}\|^{2}+\sum_{(i, j, k) \in \mathcal{I}} s_{i, j, k}(\mathbf{c}) g_{i, j, k}(\mathbf{c}),
$$

that is, $\gamma \geq\|\mathbf{a}\|^{2}$ for any c satisfying the image constraint $\left\|\mathbf{p}-\mathbf{p}^{*}\right\|_{\infty}<\delta$. From (9), we conclude that $\bar{e}_{R} \geq e_{R}$. Finally, we similarly prove that $\bar{e}_{T} \geq e_{T}$ by observing that

$$
\left(1+\|\mathbf{a}\|^{2}\right)^{2} \gamma-\|\mathbf{b}\|^{2}=\left(1+\|\mathbf{a}\|^{2}\right)^{2}\left(\gamma-\|\mathbf{t}\|^{2}\right) .
$$

Theorem 1 shows how upper bounds of $e_{R}$ and $e_{T}$ can be computed through the minimizations (25), (26), and (27) which are convex optimization constrained by linear matrix inequalities

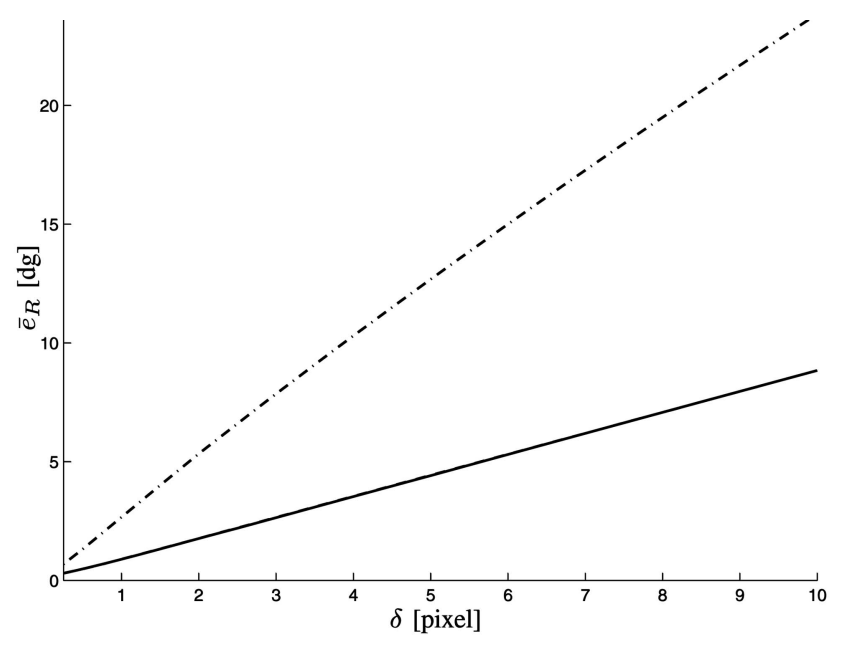

(a)

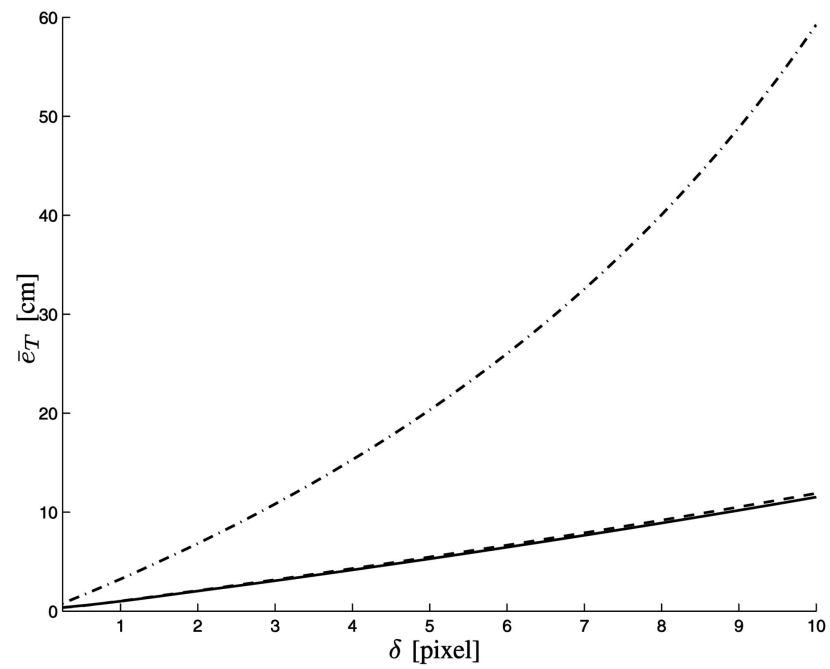

(b)

Fig. 4. Example. (a) Upper bounds $\bar{e}_{R}$ for the case of a camera able to rotate only and (b) $\bar{e}_{T}$ for the case of a camera able to translate only. The solid line is obtained by using all the nine points, the dashed line by using the first seven points only, and the dashdot line by using the first four points only.

(LMIs) known as eigenvalue problem (EVP) for which powerful tools have been recently developed (see [1] for details about EVPs). Another advantage of the proposed technique is that Theorem 1 still provides upper bounds of $e_{R}$ and $e_{T}$ even by reducing the number of free parameters in the EVP. This can be useful in order to obtain lighter optimizations, clearly at the expense of the conservativeness.

\section{EXAMPLE}

Fig. 2a shows the camera in the desired position observing three dices. The centers of the white large points in the " 4, " " 3, " and " 2 " sides are selected as features to position the camera in the teachingby-showing approach. Fig. $2 b$ shows the features in the desired view, each enclosed in a square of edge $2 \delta$ indicating the global image error in the problems (3) and (4). The question we want to answer to is: Which is the worst-case camera positioning error we might commit for this object-camera configuration when the distance between current and desired view satisfies $\left\|\hat{\mathbf{p}}-\hat{\mathbf{p}}^{*}\right\|_{\infty}<$ $\delta_{S}$ or, in other words, $\left\|\mathbf{p}-\mathbf{p}^{*}\right\|_{\infty}<\delta$ for $\delta=\delta_{S}+2 \delta_{N}$ ?

We have computed the upper bounds $\bar{e}_{R}$ and $\bar{e}_{T}$ in Theorem 1 by selecting auxiliary polynomials $s_{i, j, k}(\mathbf{c})$ of degree $2 m_{s}=2$. 
TABLE 1

Total Number of Scalar Parameters Involved in the Optimizations (25), (26), and (27)

\begin{tabular}{c|c|c|c} 
dof $\backslash n$ & 4 & 7 & 9 \\
\hline 3 & 181 & 301 & 381 \\
\hline 6 & 645 & 981 & 1205
\end{tabular}

Figs. $3 \mathrm{a}$ and $3 \mathrm{~b}$ show the results obtained for a range of values of $\delta$ in the case of full motion (six dof). Figs. $4 \mathrm{a}$ and $4 \mathrm{~b}$ show the results obtained by considering that the camera has only three dof. In particular, Fig. 4a considers a camera able to rotate only, achieved by setting $\mathbf{b}=\mathbf{0}_{3}$ from (17) to (25). Fig. $4 \mathrm{~b}$ considers a camera able to translate only, achieved by similarly setting $\mathbf{a}=\mathbf{0}_{3}$.

Table 1 shows the total number of scalar parameters involved in the optimizations (25), (26), and (27). Let us observe that, in the case of three dof, $\sigma(m)=\frac{(m+3) !}{(m ! 3 !)}$.

\section{Conclusion}

In order to design robust visual servo systems, upper bounds of the worst-case camera positioning error induced by image noise are required for a given object-camera configuration. While standard optimization tools provide lower bounds only due to the presence of local maxima, in this paper, it has been shown that upper bounds to the rotation and translation worst-case error for a certain UBB image noise intensity can be obtained through convex optimizations. These upper bounds allow one not only to quantify the maximum positioning error, but also to select optimal configurations for achieving better accuracy.

\section{ACKNOWLEDGMENTS}

The authors would like to thank the associate editor and the reviewers for their useful and constructive comments.

\section{REFERENCES}

[1] S. Boyd, L. El Ghaoui, E. Feron, and V. Balakrishnan, Linear Matrix Inequalities in System and Control Theory. SIAM, 1994.

[2] G. Chesi, A. Garulli, A. Tesi, and A. Vicino, "Solving Quadratic Distance Problems: An LMI-Based Approach," IEEE Trans. Automatic Control, vol. 48, no. 2, pp. 200-212, 2003.

[3] G. Chesi, A. Tesi, A. Vicino, and R. Genesio, “On Convexification of Some Minimum Distance Problems," Proc. Fifth European Control Conf., 1999.

[4] G. Chesi and A. Vicino, "Visual Servoing for Large Camera Displacements," IEEE Trans. Robotics, vol. 20, no. 4, pp. 724-735, 2004.

[5] P.I. Corke and S.A. Hutchinson, "A New Partitioned Approach to ImageBased Visual Servo Control," IEEE Trans. Robotics and Automation, vol. 17, no. 4, pp. 507-515, 2001.

[6] N.J. Cowan, J.D. Weingarten, and D.E. Koditschek, "Visual Servoing via Navigation Functions," IEEE Trans. Robotics and Automation, vol. 18, no. 4, pp. 521-533, 2002.

[7] K. Deguchi, “Optimal Motion Control for Image-Based Visual Servoing by Decoupling Translation and Rotation," Proc. Int'l Conf. Intelligent Robots and Systems, pp. 705-711, 1998.

[8] K. Hashimoto, "A Review on Vision-Based Control of Robot Manipulators," Advanced Robotics, vol. 17, no. 10, pp. 969-991, 2003.

[9] V. Kyrki, D. Kragic, and H.I. Christensen, "Measurement Errors in Visual Servoing," Proc. IEEE Int'l Conf. Robotics and Automation, pp. 1861-1867, 2004.

[10] E. Malis, “Visual Servoing Invariant to Changes in Camera-Intrinsic Parameters," IEEE Trans. Robotics and Automation, vol. 20, no. 1, pp. 72-81, 2004.

[11] E. Malis, F. Chaumette, and S. Boudet, "2 1/2 D Visual Servoing," IEEE Trans. Robotics and Automation, vol. 15, no. 2, pp. 238-250, 1999.

[12] Y. Mezouar and F. Chaumette, "Path Planning for Robust Image-Based Control," IEEE Trans. Robotics and Automation, vol. 18, no. 4, pp. 534-549, 2002.

[13] R.M. Murray, Z. Li, and S.S. Sastry, A Mathematical Introduction to Robotic Manipulation. CRC Press, 1994.

[14] P. Oh and P. Allen, "Visual Servoing by Partitioning Degrees-of-Freedom," IEEE Trans. Robotics and Automation, vol. 17, no. 1, pp. 1-17, 2001.

[15] A.C. Sanderson, L.E. Weiss, and C.P. Neuman, "Dynamic Sensor-Based Control of Robots with Visual Feedaback," IEEE Trans. Robotics and Automation, vol. 3, pp. 404-417, 1987.

[16] O. Tahri and F. Chaumette, "Image Moments: Generic Descriptors for Decoupled Image-Based Visual Servo," Proc. IEEE Int'l Conf. Robotics and Automation, pp. 1185-1190, 2004.

[17] C.J. Taylor and J.P. Ostrowski, "Robust Vision-Based Pose Control," Proc. IEEE Int'l Conf. Robotics and Automation, pp. 2734-2740, 2000.

[18] H. Zhang and J.P. Ostrowski, "Visual Motion Planning for Mobile Robots," IEEE Trans. Robotics and Automation, vol. 18, no. 2, pp. 199-208, 2002.

$\triangleright$ For more information on this or any other computing topic, please visit our Digital Library at www.computer.org/publications/dlib. 